\title{
Potential role of macrophage migration inhibitory factor in the pathogenesis of Marek's disease
}

\author{
Zhongjun Fan, Huanli Wang, \\ Jiahao Pan, Shupei Yu, Wenlong Xia \\ College of Marine and Biological Engineering, \\ Yancheng Teachers' University, Yancheng 224002, China \\ yctuxwl@foxmail.com
}

Received: July 9, 2019

Accepted: January 20, 2020

\begin{abstract}
Introduction: Marek's disease virus (MDV) can cause malignant T-cell lymphomas and immunosuppression in chickens. Macrophage migration inhibitory factor (MIF) not only plays a critical role in inhibiting T-cell responses, but also contributes to multiple aspects of tumour progression. The aim of this study was to reveal the potential role of MIF in the pathogenesis of MDV infection. Material and Methods: MIF gene expression levels were measured by using real-time PCR. Expression was assayed at different times in chicken embryo fibroblast (CEF) cells and tissue samples of SPF chickens infected with different MDV strains and fold change was calculated by the $2^{-} \triangle \triangle \mathrm{CT}$ method. Results: The expression of MIF was significantly downregulated $(\mathrm{p}<0.05$ and $\mathrm{FC}>2)$ in CEF cells infected with the very virulent MDV RB1B strain at $48 \mathrm{~h}$ post infection (hpi) and in the skin and spleen at 14 days post infection (dpi). The reduction of MIF expression was also found in CEF cells infected by reticuloendotheliosis virus (REV), avian leukosis virus subgroup J (ALV-J), and MDV vaccine strain CVI988 or in HD11 cells stimulated with TLR2, 3, 4, and 7 ligands. Interestingly, MIF expression decreased continuously from 7 to 28 dpi in the thymus after RB1B virus infection while it increased after CVI988 virus infection. Upregulated expression of MIF was found in CEF infected with RB1B at 96 hpi and in the spleen and skin at 21 and 28 dpi. Conclusion: The present study revealed the different expression pattern of MIF in response to MDV infection and indicated that MIF level may be associated with MDV pathogenesis.
\end{abstract}

Keywords: Marek's disease virus, macrophage migration inhibitory factor, chicken embryo fibroblasts, pathogenesis.

\section{Introduction}

Marek's disease (MD) is a lymphoproliferative disease of birds caused by a highly oncogenic, cellassociated $\alpha$-herpesvirus termed Marek's disease virus (MDV) (3). This virus can cause malignant T-cell lymphomas and immunosuppression in chickens. The primary target cells for virus infection in the chicken are B lymphocytes. The virus destroys the cells in a few days after infection and then enters a latent phase. During latent infection of activated $\mathrm{T}$ cells, expressed genes are low in abundance, but the virus can be obtained from the lymphocytes (21). These latently infected $\mathrm{T}$ lymphocytes are the means of virus dissemination to the skin and feather follicle epithelial cells. Toll-like receptors (TLRs) have key roles in the recognition of pathogens and the initiation of the innate immune response that subsequently primes the specific adaptive immune response during infection. In addition, the activation of TLRs not only has implications for antiviral defence but also contributes to tumour suppression. Increased expression of TLR2, TLR3, TLR4, and TLR7 was found in MDV-infected chicken tissues (10).

Macrophage migration inhibitory factor (MIF) is a classic pro-inflammatory cytokine secreted by several cell types, including activated $\mathrm{T}$ lymphocytes and macrophages, and plays a central role in the control of the host inflammatory and immune response (4). MIF was initially described as a soluble mediator secreted by activated $T$ cells that inhibits the migration of macrophages. MIF antibody treatment has been shown to elicit a significant increase in cytotoxic $\mathrm{T}$ lymphocyte (CTL) response, as well as increased 
levels of interferon gamma (IFN- $\gamma$ ) expression (1). MIF not only plays a critical role in inhibiting $\mathrm{T}$-cell responses but also contributes to multiple aspects of tumour progression through modulating several important biological mechanisms and processes (19). In addition, mounting evidence suggests that inflammation is closely associated with many types of cancer and MIF is a potent molecular link between inflammation and cancer (16). Moreover, MIF antibody treatment effectively suppressed tumour growth and tumour-associated angiogenesis (23). Taken together, these actions of MIF define it as important for the development and progression of cancer and render it exploitable as a marker for tumour detection.

In the previous study, MIF was identified as a differentially expressed protein in chicken thymus infected with the very virulent MDV RB1B strain, suggesting this protein might be involved in the pathogenesis of Marek's disease in poultry (9). Functional characterisation of avian MIF demonstrated the inhibition of macrophage migration, similarly to mammalian MIF, and the mediation of inflammatory responses during antigenic stimulation (12). However, there was no further investigation of its role in the pathogenesis of MDV infection or tumour progression in birds, and little is known about whether MIF is associated with the TLR-mediated immune response. In this study, we explore the potential role of MIF in the pathogenesis of MDV and make an attempt to identify the areas where knowledge is lacking in this field.

\section{Material and Methods}

Animals, cells and viruses. All chickens used in this study were one-day-old specific-pathogen-free (SPF) white Leghorns obtained from Merial Vital (Laboratory Animal Technology Co., Beijing, China). The chickens were housed in an isolation facility at the College of Marine and Biological Engineering, Yancheng Teachers' University. The RB1B (passage 15) strain of very virulent MDV and MDV vaccine strain CVI988 were maintained in the cell engineering laboratory in College of Marine and Biological Engineering, Yancheng Teachers' University.

Primary chicken embryo fibroblast (CEF) cells were prepared by standard methods from ten-day-old SPF embryos. The cells were grown in Dulbecco's modified Eagle's medium (DMEM; Gibco, Grand Island, NY, USA) supplemented with $10 \%$ foetal bovine serum (FBS) and antibiotics $(100 \mathrm{U} / \mathrm{mL}$ of penicillin and $100 \mathrm{U} / \mathrm{mL}$ of streptomycin, Gibco) and were incubated at $37^{\circ} \mathrm{C}$ in $5 \% \mathrm{CO}_{2}$ for $24 \mathrm{~h}$. After incubation, secondary $\mathrm{CEF}$ was used for virus infection. HD11, an avian macrophage cell line, was cultured in RPMI 1640 medium (Gibco) supplemented with $10 \%$ FBS, $10 \%$ tryptose phosphate broth (SigmaAldrich, St. Louis, MO, USA) and antibiotics (100
$\mathrm{U} / \mathrm{mL}$ of penicillin and $100 \mathrm{U} / \mathrm{mL}$ of streptomycin, Gibco) at $41{ }^{\circ} \mathrm{C}, 5 \% \mathrm{CO}_{2}$ and $95 \%$ humidity.

Infection of CEF cells with MDV, reticuloendotheliosis virus (REV), or avian leukosis virus subgroup (ALV-J). MDV infections of CEF cells were conducted as previously reported (14). Secondary CEF cells were seeded on 6-well plates in DMEM with $5 \% \mathrm{FBS}$ at $37^{\circ} \mathrm{C}, 5 \% \mathrm{CO}_{2}$, and $95 \%$ humidity. After $24 \mathrm{~h}$ incubation, the cells were infected with two MDV strains (RB1B and CVI988) separately at 0.1 multiplicity of infection (MOI) and each virus infected three wells on six-well plates. Then the cells were collected at 24, 48, 72 and $96 \mathrm{~h}$ post infection (hpi). REV and ALV-J infections of CEF cells were conducted by the same method, and the infected cells were collected at 24, 72, 120, and $168 \mathrm{hpi}$.

TLR stimulation experiments. HD11 cells were seeded on six-well plates and incubated for $4-16 \mathrm{~h}$ in medium containing selected TLR ligands (all from InvivoGen, Hong Kong, China) used at a unified concentration of $10 \mu \mathrm{g} / \mathrm{mL}$. The stimulants were $E$. coli 0111:B4 ultrapure TLR2 ligand peptidoglycan (PGNEB), dsRNA poly analogue synthetic TLR3 ligand (I:C) of a high molecular weight, E. coli 0111:B4 ultrapure TR4 ligand lipopolysaccharide (LPS-EB), and small synthetic antiviral molecule Imiquimod TLR7 ligand (R837). After incubation, HD11 cells were collected at $4,8,12$, and $16 \mathrm{~h}$.

Experimental animals. This experimental work was performed as reported previously (9). Briefly, 72 one-day-old chickens were randomly divided into three equal groups (an RB1B-infected group, a CVI988infected group, and a control group). The chickens were kept in separate units under similar environmental conditions. The chickens in infected groups received an intraperitoneal inoculation of $0.5 \mathrm{ml}$ of $\mathrm{RB} 1 \mathrm{~B}$ or CVI988 virus solution at a dose of 2,000 plaqueforming units. At 7, 14, 21, and $28 \mathrm{dpi}$, nine chickens (three RB1B-infected, three CVI988-infected, and three uninfected control birds) were sacrificed and samples of the thymus, spleen, bursa, and skin were excised rapidly, rinsed with ice cold $\mathrm{PBS}$ to remove blood contaminants and immediately stored in liquid nitrogen until the real-time PCR analyses.

RNA isolation and quantitative real-time PCR. These procedures were conducted by methods previously reported (8). Briefly, total RNA was extracted from each tissue $(0.1 \mathrm{~g})$ or cell sample $(2.5 \times$ $10^{6}$ per well) using an AxyPrep Multisource Total RNA Miniprep Kit (Axygen, Union City, CA, USA), and each RNA sample $(1 \mu \mathrm{g})$ was reversed into first-strand cDNA using the PrimeScript RT Master Mix (TaKaRa Biomedical Technology, Beijing, China) following the manufacturer's instructions. Then the cDNA was diluted with nuclease-free water to $1: 10$, and $1 \mu \mathrm{L}$ of the diluted sample was used for the real-time PCR with $400 \mathrm{nM}$ of primers and $10 \mu \mathrm{L}$ of SYBR Green Master Mix (TaKaRa) in a final volume of $20 \mu \mathrm{L}$. The primer sequences for MIF, GAPDH (12), and 18S rRNA (15) 
have been previously reported and are given in Table 1. The expression level of MIF was determined using real-time PCR (7500 Real-Time PCR System, Applied Biosystems, Foster City, CA, USA) with amplification conditions of $95^{\circ} \mathrm{C}$ for $30 \mathrm{~s}, 40$ cycles of $95^{\circ} \mathrm{C}$ for $5 \mathrm{~s}$, and $60^{\circ} \mathrm{C}$ for $34 \mathrm{~s}$. The expression level of MIF was normalised against the expression of chicken GAPDH in tissues or 18S mRNA in CEF cells and fold change in gene expression was calculated by the $2^{-\triangle \triangle \mathrm{CT}}$ method.

Statistical analysis. Statistical analysis was conducted with the Statistical Package for the Social Sciences in version 16.0 (IBM, Armonk, NY, USA). Student's $t$-test was used to determine significant difference between fold change values of control and infected or treated MIF genes. Fold changes (FC) that had both $\mathrm{P}<0.05$ and greater than two-fold difference in expression levels were considered significantly different. Standard error was calculated using the FC values of three replicates for each gene measured.

\section{Results}

Differential expression pattern of MIF in chicken fibroblasts (CEF). To analyse the potential role in the pathogenesis of MDV infection, we first detected the expression of MIF in CEF cells infected with two MDV strains (RB1B and CVI988). We found that the expression of MIF was obviously upregulated at $24 \mathrm{hpi}$ and significantly down regulated $(\mathrm{P}<0.001$ and $\mathrm{FC}>2$ ) at $48 \mathrm{hpi}$ in CEF cells infected with the RB1B strain. After 48 hpi, the expression trend of MIF was gradually rising and it was significantly upregulated $(\mathrm{P}<0.01$ and $\mathrm{FC}>2)$ at 96 hpi. Unlike in RB1B infection, MIF expression sharply reduced in CVI988 strain-infected-CEF cells at $24 \mathrm{hpi}$ and then significantly augmented $(\mathrm{P}<0.01$ and $\mathrm{FC}>2)$ at 72 and 96 hpi (Fig. 1A). To further confirm whether MIF induction is a unique characteristic for MDV infection in three avian viruses which induced immunosuppressive and tumour diseases in poultry (MDV, REV, and ALV), we next detected the expression of MIF in CEF cells infected with REV or ALV-J. Interestingly, MIF was not induced by REV or ALV-J and both reduced MIF expression after infection (Fig. 1B). MIF was actually significantly decreased by $\mathrm{REV}$ at $72(\mathrm{P}<0.001$ and $\mathrm{FC}>2)$ and $120 \mathrm{hpi}$ $(\mathrm{P}<0.01$ and $\mathrm{FC}>2)$.

Differential expression pattern of MIF in chicken avian macrophage cell line (HD11). To investigate whether MIF is associated with the expressions of TLRs, we detected the expression levels of MIF in HD11 cells treated with TLR ligands. We found that TLR2 and TLR4 stimulation elicits a continuous and gradual reduction of MIF expression from 4 to $16 \mathrm{~h}$ while there is a $4 \mathrm{~h}$ delay for TLR3 and TLR7 stimulation (Fig. 1C and D).
A

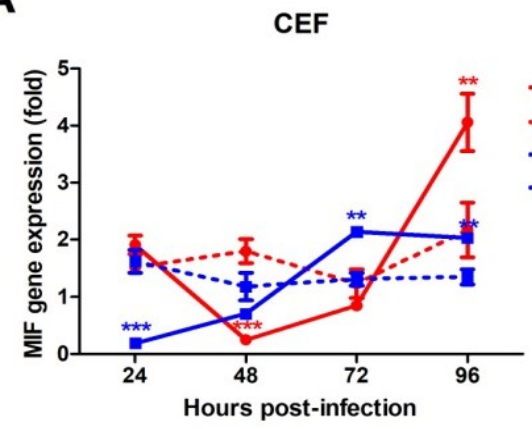

C

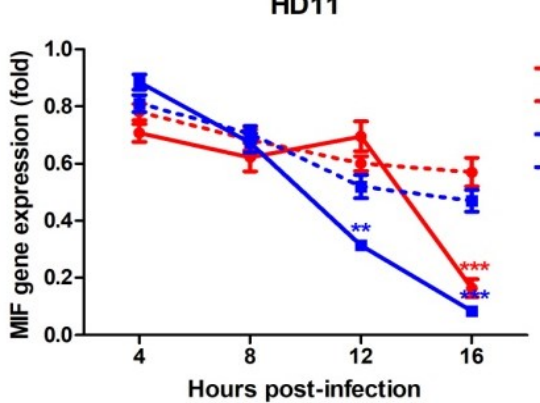

B
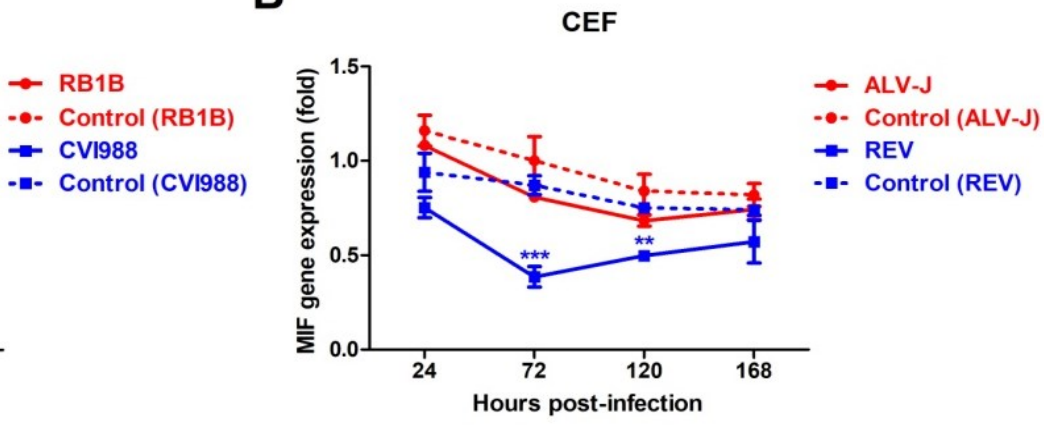

D

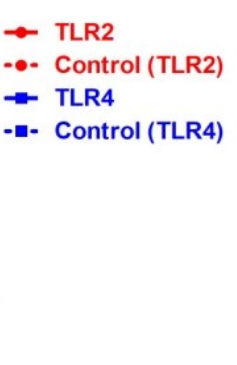

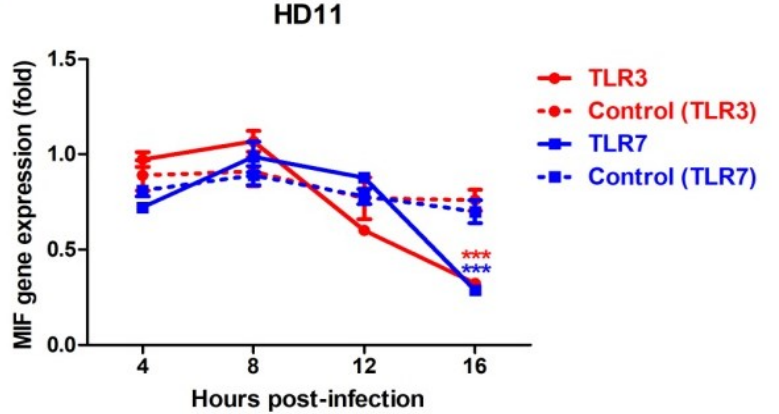

Fig. 1. MIF expression in CEF and HD11 cells. A - expression of MIF in CEF cells infected with RB1B or CVI988 strain; B - expression of MIF in chicken fibroblasts infected with REV or ALV-J; C - expression of MIF in HD11 in response to TLR2 and four stimulations; D - expression of MIF in CEF in response to TLR3 and TLR7 stimulations. The different number of asterisks (*) indicates statistically significant difference for the comparison of control (uninfected or untreated) and infected (or stimulated) transcripts at the same time point as determined by Student's $t$-test. $* *-\mathrm{P}<0.01, * * *-\mathrm{P}<0.001$. Error bars represent standard error 
Table 1. Primers used for real-time PCR

\begin{tabular}{llll}
\hline Gene & Primer Sequence $\left(5^{\prime}-3^{\prime}\right)$ & Product size $(b p)$ & Accession number \\
\hline MIF & F: GCCCGCGCAGTACATAGC & 57 & XM42_5824 \\
& R: CCCCCGAAGGACATCATCT & & NM_204305 \\
GAPDH & F: AGGGTGGTGCTAAGCGTGTTA & 78 & \\
& R: TCTCATGGTTGACACCCATCA & & AF173612 \\
& F: TCAGATACCGTCGTAGTTCC & 154 & \\
\hline
\end{tabular}

A

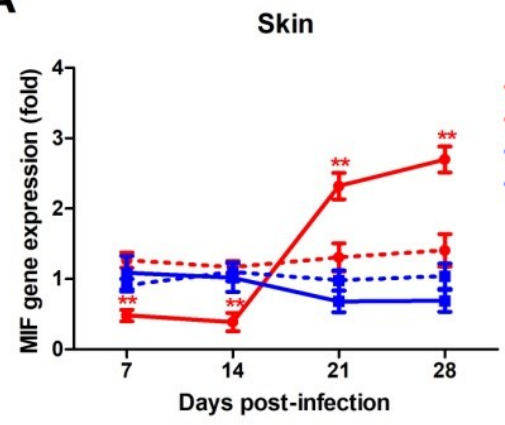

C

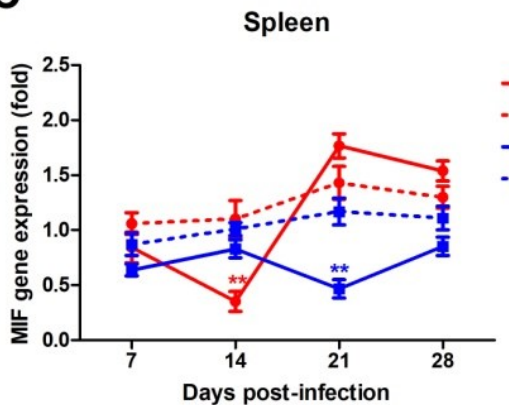

B

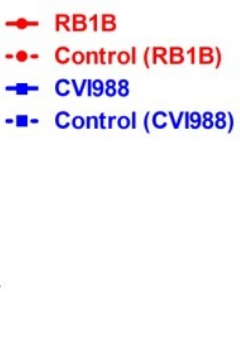

D

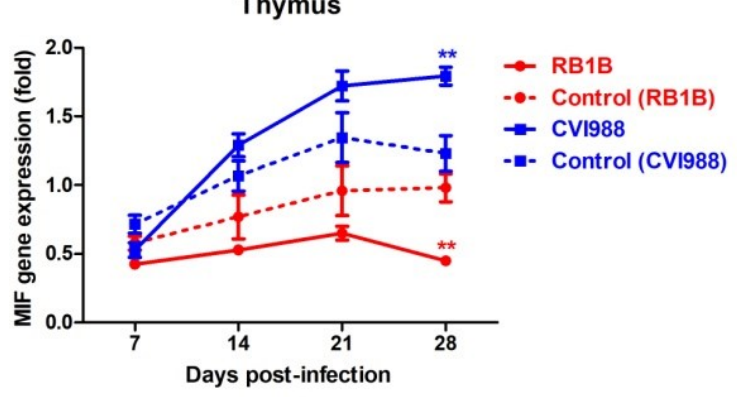

Bursa of Fabricius

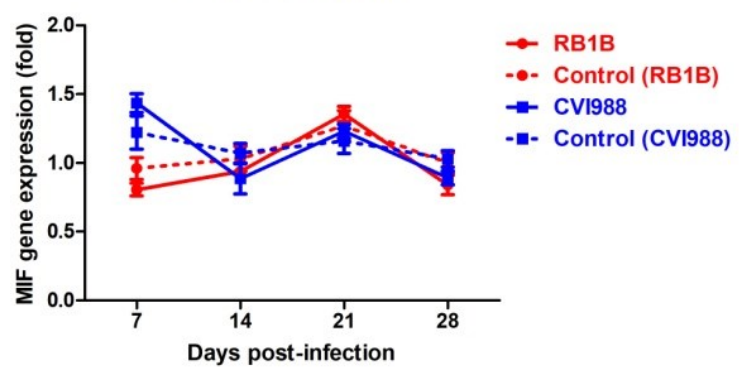

Fig. 2. MIF expression in chicken skin infected with RB1B or CVI988 strain. The different number of asterisks (*) indicates statistically significant difference for the comparison of control (uninfected) and infected transcripts at the same time point as determined by Student's $t$-test. $* *-\mathrm{P}<0.01$. Error bars represent standard error

Differential expression pattern of MIF in chicken tissues. MIF expression was significantly diminished $(\mathrm{P}<0.01$ and $\mathrm{FC}>2)$ in RB1B-infected chicken skin at 7 and $14 \mathrm{dpi}$ and elevated at 21 and $28 \mathrm{dpi}$, whereas it did not show any significant change in CVI988-infected chicken skin at the same time points (Fig. 2A). Reduced expression of MIF was also observed in RB1B-infected chicken thymus at four time points while the MIF expression trend was persistently increased from 7 to $28 \mathrm{dpi}$ in CVI988infected chicken thymus (Fig. 2B). In the spleen, MIF expression was slightly reduced by CVI988 infection and significantly downregulated $(\mathrm{P}<0.01$ and $\mathrm{FC}>2)$ at $21 \mathrm{dpi}$. MIF expression level was also significantly decreased $(\mathrm{P}<0.01$ and $\mathrm{FC}>2)$ by RB1B infection at $14 \mathrm{dpi}$ while obviously upregulated at 21 and 28 dpi (Fig. 2C). In addition, we did not find any significant change of MIF expression in chicken bursa of Fabricius infected with RB1B or CVI988 strains (Fig. 2D).

\section{Discussion}

Despite our knowledge of molecular and cellular mechanisms of immunity against MD, we still have a limited understanding of the process and dynamics of T-cell mediated responses to the virus. Moreover, significant information on critical aspects of virus latency in lymphoid cells and the virus-host interaction in MDV-induced lymphoma is lacking. Importantly, there is little known about the molecular determinants of the host which govern T-lymphocyte immune response and transformation in latent MDV infection. T-lymphocytes are of key importance to the immune system and are at the core of adaptive immunity, thus the virus is not sufficient by itself for induction of T-cell lymphomas and the regulatory mechanisms of T-cell immunity could be employed by MDV. Reports showed that MDV influences the expression of genes associated with $\mathrm{T}$ lymphocyte responses during MDV infection (13). MIF plays a critical role in inhibiting T-cell responses, and has assumed a centrally important 
mediatory function for innate immunity. In this study, we have further revealed the different expression pattern of chicken MIF in response to MDV infection and discussed in detail the potential role of this factor in the course of MDV infection.

MIF has emerged as a pivotal mediator of innate immunity (4). This protein modulates not only macrophage but also $\mathrm{T}$ cell functions (4), and especially exerts significant effects on regulation of anti-tumour and antigen-specific cytotoxic T-lymphocyte responses. The downregulation of the MIF gene in avian cells reflects the host immune response to virus infection or TLR stimulations. In fact, MIF expression was also decreased during early MDV infection, as MIF showed reduction in CEF at $24 \mathrm{hpi}$ or $48 \mathrm{hpi}$ and in chicken tissues at $7 \mathrm{dpi}$ or $14 \mathrm{dpi}$. In addition, we also observed a continuous and gradual reduction of MIF expression in the avian HD11 macrophage cell line after a cell response was elicited by TLR stimulation, indicating that MIF in immune cells can be affected by TLR status. Studies in vivo showed that neutralisation of MIF can promote cytotoxic T-lymphocyte activity, increase expression levels of IFN- $\gamma$, and increase T lymphocyte homing to sites of tumour invasion (1), while expression of MIF leads to the inhibition of antitumour $\mathrm{T}$ lymphocyte reactivity (25) and $\mathrm{T}$ lymphocyte activation (24). A possible theory is that high MIF levels cause activation-induced T-cell death through an IFN- $\gamma$ pathway and may eliminate activated $\mathrm{T}$ cells from the tumour microenvironment and thus facilitate the tumour's evasion of immune surveillance (24). Interestingly, the very virulent and oncogenic RB1B strain causes reduced expression of MIF in an infected chicken thymus while the non-oncogenic CVI988 vaccine strain causes increased expression. At present, there is little reported research into the function of chicken MIF, and we deduced that this protein plays either a protective or deleterious role in the immune response to different pathogens. Increased expression of MIF after latent MDV infection could inhibit T-cell responses.

Increased expression of MIF in the spleen at 21 and $28 \mathrm{dpi}$ and in CEF at $96 \mathrm{hpi}$ could be relevant to MDV RB1B strain infection and replication, while in skin at 21 and 28 dpi it might be associated with the production of MDV virus particles. Firstly, we observed that the expression trend of MIF was gradually rising along with the replication of MDV after 48 hpi for RB1B or 24 hpi for CVI988 and was significantly up regulated at 72 and/or $96 \mathrm{hpi}$. The results suggested that MIF expression was influenced by MDV during the different stages of pathogenesis. However, MIF was not induced by the other two of the three avian viruses (ALV-J and REV) which induced immunosuppressive and tumourigenic diseases in poultry in infected CEF cells. This may indicate a direct role for MIF in MDV replication or pathogenesis. Induction of MIF expression was also found in herpes simplex virus type 1 (HSV-1) (18), human cytomegalovirus (HCMV), (6) and dengue virus
(5). HCMV paralyses macrophage motility through release of MIF (6), and MIF promotes HIV-1 replication through the activation of HIV-1 long terminal repeats (LTR) (22).

However, the reduction of MIF in skin, spleen, and thymus during early and latent infection may promote MDV spread. This is because strong macrophage migration activity when MIF is reduced will not only enhance random migration of macrophages but elicit $\mathrm{T}$ lymphocyte activation, and this could offer an opportunity for macrophages that carry MDV to spread the virus to T lymphocytes, and then latently infected lymphocytes can disseminate the virus to different sites. Indeed, infection of macrophages in vivo by MDV has been reported (2). Some MDV strains can replicate in macrophages, which leads to increased macrophage death (2) and a heavy infiltration of macrophages occurs around blood vessels at 8-10 days post MDV infection (7). In addition, macrophages play an essential role in the sensing and elimination of invasive microorganisms and this also offers the better option for virus contact with other immune cells. Thus, macrophages are excellent candidates for transporting MDV to primary lymphoid organs during the earliest stages of pathogenesis, and the reduction of MIF enhanced macrophage migration might be a potential mechanism employed by MDV to increase virus transport.

In the chicken spleen and skin infected with the very virulent and oncogenic strain $\mathrm{RB} 1 \mathrm{~B}$, we observed the increased expression of MIF gene at 21 and 28 dpi. However, MIF expression was reduced in the nononcogenic vaccine strain CVI988-infected spleen and in skin its expression did not show any significant change at the same time points. These findings suggested that MIF might be employed by MDV to induce lymphoma occurrence. Firstly, MIF sustains macrophage survival and function by suppressing p53dependent apoptosis (17) and this is important for MDV-infected macrophage to spread the virus. Secondly, MIF exerts significant pro-tumour effects by regulation of anti-tumour T-lymphocyte responses. Host T-lymphocyte immunity is the biggest obstacle for MDV infection and the virus is not sufficient by itself for induction of T-cell lymphomas. MIF plays a dual role in both inhibiting $\mathrm{T}$-cell responses and promoting tumour cell growth, and thus this regulatory mechanism could be employed by MDV to promote lymphoma occurrence. More importantly, MIF has been shown to mediate several important biological mechanisms and processes by which tumours thrive and spread. One of these mechanisms is the negative regulation of the important p53 tumour suppressor pathway (11), and the other is the modulation of hypoxic adaptation within the tumour microenvironment through the direct promotion of hypoxia-induced stabilisation of HIF-1 $\alpha$ (20). However, the contributions of MIF to MDV-specific T-cell immunity and the mechanism of MD lymphoma occurrence need further investigation. 
In summary, the present results provide the different expression pattern of MIF gene in response to the very virulent RB1B strain and CVI988 vaccine strain infections and might reveal a potential role of MIF in the pathogenesis of MDV infection. MIF might be a mechanism employed by MDV to increase virus replication and transport and promote MD lymphoma occurrence and evolution.

Conflict of Interests Statement: The authors declare that there is no conflict of interests regarding the publication of this article.

Financial Disclosure Statement: This research was supported by the Guiding Plan for Agricultural Science and Technology of Yancheng City (YKN2013014).

Animal Rights Statement: The animal experiments were conducted in accordance with the guidelines provided by the Chinese Council on Animal Care. All experiments complied with institutional animal care guidelines and were approved by the Animal Ethics Committee of Yancheng Teachers' University.

\section{References}

1. Abe R., Peng T., Sailors J., Bucala R., Metz C.N.: Regulation of the CTL response by macrophage migration inhibitory factor. J Immunol 2001, 166, 747-753.

2. Barrow A.D., Burgess S.C., Baigent S.J., Howes K., Nair V.K.: Infection of macrophages by a lymphotropic herpesvirus: a new tropism for Marek's disease virus. J Gen Virol 2003, 84, 2635-2645.

3. Biggs P.M.: The history and biology of Marek's disease virus. Curr Top Microbiol Immunol 2001, 255, 1-24.

4. Calandra T., Roger T.: Macrophage migration inhibitory factor: a regulator of innate immunity. Nat Rev Immunol 2003, 3, 791-800.

5. Chuang Y.C., Lei H.Y., Liu H.S., Lin Y.S., Fu T.F., Yeh T.M.: Macrophage migration inhibitory factor induced by dengue virus infection increases vascular permeability. Cytokine 2011, 54, 222-231.

6. Frascaroli G., Varani S., Blankenhorn N., Pretsch R., Bacher M., Leng L., Bucala R., Landini M.P., Mertens T.: Human cytomegalovirus paralyzes macrophage motility through downregulation of chemokine receptors, reorganization of the cytoskeleton, and release of macrophage migration inhibitory factor. J Immunol 2009, 182, 477-488.

7. Gimeno I.M., Witter R.L., Hunt H.D., Lee L.F., Reddy S.M., Neumann U.: Marek's disease virus infection in the brain: virus replication, cellular infiltration, and major histocompatibility complex antigen expression. Vet Pathol 2001, 38, 491-503.

8. Hu X., Qin A., Miao J., Xu W., Yu C., Qian K., Shao H.: Transcriptional profile of Marek's disease virus genes in chicken thymus during different phases of MDV infection. Arch Virol 2013, 158, 1787-1793.

9. Hu X., Qin A., Qian K., Shao H., Yu C., Xu W., Miao J.: Analysis of protein expression profiles in the thymus of chickens infected with Marek's disease virus. Virol J 2012, 9, 1-18.
10. Hu X., Xu W., Qin A., Wu G., Qian K., Shao H., Ye J.: Marek's disease virus may interfere with $\mathrm{T}$ cell immunity by TLR3 signals. Vet Res Commun 2014, 38, 149-156.

11. Jung H., Seong H.A., Ha H.: Critical role of cysteine residue 81 of macrophage migration inhibitory factor (MIF) in MIF-induced inhibition of p53 activity. J Biol Chem 2008, 283, 20383-20396.

12. Kim S., Miska K.B., Jenkins M.C., Fetterer R.H., Cox C.M., Stuard L.H., Dalloul R.A.: Molecular cloning and functional characterization of the avian macrophage migration inhibitory factor (MIF). Dev Comp Immunol 2010, 34, 1021-1032.

13. Laursen A.M.S., Kulkarni R.R., Taha-Abdelaziz K., Plattner B.L., Read L.R., Sharif S.: Characterizaton of gamma delta T cells in Marek's disease virus (Gallid herpesvirus 2) infection of chickens. Virology 2018, 522, 56-64.

14. Levy A.M., Davidson I., Burgess S.C., Heller E.D.: Major histocompatibility complex class I is downregulated in Marek's disease virus infected chicken embryo fibroblasts and corrected by chicken interferon. Comp Immunol Microbiol Infect Dis 2003, 26, 189-198.

15. Li Y.P., Bang D.D., Handberg K.J., Jorgensen P.H., Zhang M.F.: Evaluation of the suitability of six host genes as internal control in real-time RT-PCR assays in chicken embryo cell cultures infected with infectious bursal disease virus. Vet Microbiol 2005, 110, 155-165.

16. Mantovani A., Allavena P., Sica A., Balkwill F.: Cancer-related inflammation. Nature 2008, 454, 436-444.

17. Mitchell R.A., Liao H., Chesney J., Fingerle-Rowson G., Baugh J., David J., Bucala R.: Macrophage migration inhibitory factor (MIF) sustains macrophage proinflammatory function by inhibiting p53: regulatory role in the innate immune response. Proc Natl Acad Sci USA 2002, 99, 345-350.

18. Miyazaki D., Haruki T., Takeda S., Sasaki S., Yakura K., Terasaka Y., Komatsu N., Yamagami S., Touge H., Touge C., Inoue Y.: Herpes simplex virus type 1-induced transcriptional networks of corneal endothelial cells indicate antigen presentation function. Invest Ophthalmol Vis Sci 2011, 52, 4282-4293.

19. Nobre C.C., de Araújo J.M., Fernandes T.A., Cobucci R.N., Lanza D.C., Andrade V.S., Fernandes J.V.: Macrophage migration inhibitory factor (MIF): biological activities and relation with cancer. Pathol Oncol Res 2017, 23, 235-244.

20. Oda S., Oda T., Nishi K., Takabuchi S., Wakamatsu T., Tanaka T., Adachi T., Fukuda K., Semenza G.L., Hirota K.: Macrophage migration inhibitory factor activates hypoxia-inducible factor in a p53-dependent manner. PLoS One 2008, 3, e2215.

21. Osterrieder N., Kamil J.P., Schumacher D., Tischer B.K., Trapp S.: Marek's disease virus: from miasma to model. Nat Rev Microbiol 2006, 4, 283-294.

22. Regis E.G., Barreto-de-Souza V., Morgado M.G., Bozza M.T., Leng L., Bucala R., Bou-Habib D.C.: Elevated levels of macrophage migration inhibitory factor (MIF) in the plasma of HIV-1-infected patients and in HIV-1-infected cell cultures: a relevant role on viral replication. Virology 2010, 399, 31-38.

23. Schinagl A., Thiele M., Douillard P., Völkel D., Kenner L., Kazemi Z., Freissmuth M., Scheiflinger F., Kerschbaumer R.J.: Oxidized macrophage migration inhibitory factor is a potential new tissue marker and drug target in cancer. Oncotarget 2016, 7, 73486-73496.

24. Yan X., Orentas R.J., Johnson B.D.: Tumor-derived macrophage migration inhibitory factor (MIF) inhibits $\mathrm{T}$ lymphocyte activation. Cytokine 2006, 33, 188-198.

25. Zhou Q., Yan X., Gershan J., Orentas R.J., Johnson B.D.: Expression of macrophage migration inhibitory factor by neuroblastoma leads to the inhibition of antitumor $\mathrm{T}$ cell reactivity in vivo. J Immunol 2008, 181, 1877-1886. 